\title{
GABOR SYSTEMS ON POSITIVE HALF LINE VIA WALSH-FOURIER TRANSFORM
}

\author{
AHMAD O., SHEIKH N.A.
}

\begin{abstract}
Gabor systems play a vital role not only in modern harmonic analysis but also in several fields of applied mathematics, for instances, detection of chirps, or image processing. In this paper, we investigate Gabor systems on positive half line via Walsh-Fourier transform. We provide the complete characterization of orthogonal Gabor systems on positive half line. Furthermore, we provide the characterization of tight frames and orthonormal bases of Gabor systems on positive half line in Fourier domain.

Key words and phrases: Gabor frame, Walsh-Fourier transform, orthogonality.
\end{abstract}

National Institute of Technology, 190006, Srinagar, India

E-mail: siawoahmadegmail.com (Ahmad O.), neyaznit@yahoo.co.in (Sheikh N.A.)

\section{INTRODUCTION}

In the framework of mathematical analysis and linear algebra, redundant representations are obtained by analysing vectors with respect to an overcomplete system. Then the obtained vectors are interpreted using the frame theory as introduced by R.J. Duffin and A.C. Shaeffer [4]. In signal processing, this concept has become very useful in analyzing the completeness and stability of linear discrete signal representations. Frames did not seem to generate much interest until the ground-breaking work of I. Daubechies et al. [3]. They combined the theory of continuous wavelet transforms with the theory of frames to introduce wavelet (affine) frames for $L^{2}(\mathbb{R})$. Since then the theory of frames began to be more widely investigated, and now it is found to be useful in signal processing, image processing, harmonic analysis, sampling theory, data transmission with erasures, quantum computing and medicine. Recently, more applications of the theory of frames are found in diverse areas including optics, filter banks, signal detection and in the study of Besov spaces and Banach spaces. We refer the reader to $[2,10]$ for an introduction to frame theory and its applications.

One of the central tasks in signal processing and time-frequency analysis is to find convenient series expansions of functions in $L^{2}(\mathbb{R})$. A popular choice of such series expansions is by use of Gabor frames. Gabor systems are collections of functions

$$
\mathcal{G}(a, b, \psi)=\left\{M_{m b} T_{n a} \psi(x)=: e^{2 \pi i m b x} \psi(x-n a): m, n \in \mathbb{Z}\right\},
$$

which are built by the combined action of modulations and translations of a single function, and hence, can be viewed as the set of time-frequency shifts of $\psi(x) \in L^{2}(\mathbb{R})$ along the lattice $a \mathbb{Z} \times b \mathbb{Z}$ in $\mathbb{R}^{2}$. Such systems, also called Weyl-Heisenberg systems, were introduced by Gabor [8] with the aim of constructing efficient, time-frequency localized expansions of signals as an 
infinite linear combinations of elements in (1). The system $\mathcal{G}(a, b, \psi)$ given by (1) is called a Gabor frame if there exist constants $A, B>0$ such that

$$
A\|f\|_{2}^{2} \leq \sum_{m \in \mathbb{Z}} \sum_{n \in \mathbb{Z}}\left|\left\langle f, M_{m b} T_{n a} \psi\right\rangle\right|^{2} \leq B\|f\|_{2}^{2}
$$

holds for every $f \in L^{2}(\mathbb{R})$, and we call the optimal constants $A$ and $B$ the lower frame bound and the upper frame bound, respectively. A tight Gabor frame refers to the case when $A=B$, and a normalized tight frame refers to the case when $A=B=1$. Gabor systems that form frames for $L^{2}(\mathbb{R})$ have a wide variety of applications. One of the most important problem in practice is therefore to determine conditions for Gabor systems to be frames. In practice, once the window function has been chosen, the first question to investigate for Gabor analysis is to find the values of the time-frequency parameters $a, b$ such that $\mathcal{G}(a, b, \psi)$ is a frame. Therefore, the product $a b$ will decide whether the system $\mathcal{G}(a, b, \psi)$ constitutes a frame or even complete for $L^{2}(\mathbb{R})$ or not. In this context, a useful tool is the Ron and Shen criterion (see [12]). By using this criterion, K. Gröchenig et al. [9] have proved that the system $\mathcal{G}(a, b, \psi)$ cannot be a frame for $L^{2}(\mathbb{R})$ if $|a b|>1$ and have also shown that the system $\mathcal{G}(a, b, \psi)$ will form an orthonormal basis for $L^{2}(\mathbb{R})$ if $|a b|=1$.

Gabor analysis is a pervasive signal processing method for decomposing and reconstructing signals from their time frequency projections and also in the context of speech processing, texture segmentation, pattern and object recognition. In order to analyze the dynamic time frequency samples of the signals that contain a wide range of spatial and frequency components, the resolution of which is normally very poor, the single windowed Gabor expansion is not suitable. To address this issue, one of the best choices is multigenerator Gabor system which a set of multiple windows of various time frequency localizations in frame system, the representation of signals of multiple and time-varying frequencies would have their corresponding windowing templates and resolutions to relate to. Ahmad $\mathrm{O}$. and his collaborators in the series of papers [1,15-17] investigated Gabor frames on local fields and obtained various interesting results.

There is a substantial body of work that has been concerned with the wavelet and Gabor frames on positive half line. Shah F.A. [14] constructed Gabor frame on positive half line and obtained necessary and sufficient conditions for Gabor frames in $L^{2}\left(\mathbb{R}^{+}\right)$. Recent results on wavelet frames in $L^{2}\left(\mathbb{R}^{+}\right)$can be found in $[5-7,18]$ and the references therein. Motivated and inspired by the above works, we investigate Gabor systems on positive half line via Walsh-Fourier transform. We provide the complete characterization of orthogonal Gabor systems on positive half line. Furthermore, we provide the characterization of tight frames and orthonormal bases of Gabor systems on positive half line in Fourier domain.

\section{WALSH-FOURIER ANALYSIS}

As usual, let $\mathbb{R}^{+}=[0,+\infty), \mathbb{Z}^{+}=\{0,1,2, \ldots\}$ and $\mathbb{N}=\mathbb{Z}^{+} \backslash\{0\}$. Denote by $[x]$ the integer part of $x$. Let $p$ be a fixed natural number greater than 1 . For $x \in \mathbb{R}^{+}$and any positive integer $j$, we set

$$
x_{j}=\left[p^{j} x\right](\bmod p), \quad x_{-j}=\left[p^{1-j} x\right](\bmod p)
$$


where $x_{j}, x_{-j} \in\{0,1, \ldots, p-1\}$. Clearly, $x_{j}$ and $x_{-j}$ are the digits in the $p$-expansion of $x$ :

$$
x=\sum_{j<0} x_{-j} p^{-j-1}+\sum_{j>0} x_{j} p^{-j}
$$

Moreover, the first sum on the right is always finite. Besides,

$$
[x]=\sum_{j<0} x_{-j} p^{-j-1}, \quad\{x\}=\sum_{j>0} x_{j} p^{-j},
$$

where $[x]$ and $\{x\}$ are, respectively, the integral and fractional parts of $x$.

Consider on $\mathbb{R}^{+}$the addition defined as follows:

$$
x \oplus y=\sum_{j<0} \zeta_{j} p^{-j-1}+\sum_{j>0} \zeta_{j} p^{-j}
$$

with $\zeta_{j}=x_{j}+y_{j}(\bmod p), j \in \mathbb{Z} \backslash\{0\}$, where $\zeta_{j} \in\{0,1, \ldots, p-1\}$ and $x_{j}, y_{j}$ are calculated by (2). Clearly, $[x \oplus y]=[x] \oplus[y]$ and $\{x \oplus y\}=\{x\} \oplus\{y\}$. As usual, we write $z=x \ominus y$ if $z \oplus y=x$, where $\ominus$ denotes subtraction modulo $p$ in $\mathbb{R}^{+}$.

Let $\varepsilon_{p}=\exp (2 \pi i / p)$, we define a function $r_{0}(x)$ on $[0,1)$ by

$$
r_{0}(x)= \begin{cases}1, & \text { if } x \in[0,1 / p), \\ \varepsilon_{p}^{\ell}, & \text { if } x \in\left[\ell p^{-1},(\ell+1) p^{-1}\right), \quad \ell=1,2, \ldots, p-1 .\end{cases}
$$

The extension of the function $r_{0}$ to $\mathbb{R}^{+}$is given by the equality $r_{0}(x+1)=r_{0}(x), \forall x \in \mathbb{R}^{+}$. Then, the system of generalized Walsh functions $\left\{w_{m}(x): m \in \mathbb{Z}^{+}\right\}$on $[0,1)$ is defined by

$$
w_{0}(x) \equiv 1 \quad \text { and } \quad w_{m}(x)=\prod_{j=0}^{k}\left(r_{0}\left(p^{j} x\right)\right)^{\mu_{j}}
$$

where $m=\sum_{j=0}^{k} \mu_{j} p^{j}, \mu_{j} \in\{0,1, \ldots, p-1\}, \mu_{k} \neq 0$. They have many properties similar to those of the Haar functions and trigonometric series, and form a complete orthogonal system. Further, by a Walsh polynomial we shall mean a finite linear combination of Walsh functions. For $x, y \in \mathbb{R}^{+}$, let

$$
\chi(x, y)=\exp \left(\frac{2 \pi i}{p} \sum_{j=1}^{\infty}\left(x_{j} y_{-j}+x_{-j} y_{j}\right)\right),
$$

where $x_{j}, y_{j}$ are given by (2).

We observe that

$$
\chi\left(x, \frac{m}{p^{n}}\right)=\chi\left(\frac{x}{p^{n}}, m\right)=w_{m}\left(\frac{x}{p^{n}}\right), \quad \forall x \in\left[0, p^{n}\right), m, n \in \mathbb{Z}^{+},
$$

and

$$
\chi(x \oplus y, z)=\chi(x, z) \chi(y, z), \quad \chi(x \ominus y, z)=\chi(x, z) \overline{\chi(y, z)},
$$

where $x, y, z \in \mathbb{R}^{+}$and $x \oplus y$ is $p$-adic irrational. It is well known that systems $\{\chi(\alpha, .)\}_{\alpha=0}^{\infty}$ and $\{\chi(\cdot, \alpha)\}_{\alpha=0}^{\infty}$ are orthonormal bases in $L^{2}[0,1]$ (see B.I. Golubov et al. [11]). 
The Walsh-Fourier transform of a function $f \in L^{1}\left(\mathbb{R}^{+}\right) \cap L^{2}\left(\mathbb{R}^{+}\right)$is defined by

$$
\hat{f}(\xi)=\int_{\mathbb{R}^{+}} f(x) \overline{\chi(x, \xi)} d x,
$$

where $\chi(x, \xi)$ is given by (3). The Walsh-Fourier operator $\mathcal{F}: L^{1}\left(\mathbb{R}^{+}\right) \cap L^{2}\left(\mathbb{R}^{+}\right) \rightarrow L^{2}\left(\mathbb{R}^{+}\right)$, $\mathcal{F} f=\hat{f}$, extends uniquely to the whole space $L^{2}\left(\mathbb{R}^{+}\right)$. The properties of the Walsh-Fourier transform are quite similar to those of the classic Fourier transform (see [11,13]). In particular, if $f \in L^{2}\left(\mathbb{R}^{+}\right)$, then $\hat{f} \in L^{2}\left(\mathbb{R}^{+}\right)$and

$$
\|\hat{f}\|_{L^{2}\left(\mathbb{R}^{+}\right)}=\|f\|_{L^{2}\left(\mathbb{R}^{+}\right)} .
$$

Moreover, if $f \in L^{2}[0,1]$, then we can define the Walsh-Fourier coefficients of $f$ as

$$
\hat{f}(n)=\int_{0}^{1} f(x) \overline{w_{n}(x)} d x .
$$

The series $\sum_{n \in \mathbb{Z}^{+}} \hat{f}(n) w_{n}(x)$ is called the Walsh-Fourier series of $f$. Therefore, from the standard $L^{2}$-theory, we conclude that the Walsh-Fourier series of $f$ converges to $f$ in $L^{2}[0,1]$ and Parseval's identity holds:

$$
\|f\|_{2}^{2}=\int_{0}^{1}|f(x)|^{2} d x=\sum_{n \in \mathbb{Z}^{+}}|\hat{f}(n)|^{2} .
$$

By $p$-adic interval $I \subset \mathbb{R}^{+}$of range $n$, we mean intervals of the form

$$
I=I_{n}^{k}=\left[k p^{-n},(k+1) p^{-n}\right), k \in \mathbb{Z}^{+} .
$$

The $p$-adic topology is generated by the collection of $p$-adic intervals and each $p$-adic interval is both open and closed under the $p$-adic topology (see [11]). The family $\left\{\left[0, p^{-j}\right): j \in \mathbb{Z}\right\}$ forms a fundamental system of the $p$-adic topology on $\mathbb{R}^{+}$. Therefore, the generalized Walsh functions $w_{j}(x), 0 \leq j \leq p^{n}-1$, assume constant values on each $p$-adic interval $I_{n}^{k}$ and hence continuous on these intervals. Thus, $w_{j}(x)=1$ for $x \in I_{n}^{0}$.

Let $\mathcal{E}_{n}\left(\mathbb{R}^{+}\right)$be the space of $p$-adic entire functions of order $n$, that is, the set of all functions, which are constant on all $p$-adic intervals of range $n$. Thus, for every $f \in \mathcal{E}_{n}\left(\mathbb{R}^{+}\right)$, we have

$$
f(x)=\sum_{k \in \mathbb{Z}^{+}} f\left(p^{-n} k\right) \chi_{I_{n}^{k}}(x), \quad x \in \mathbb{R}^{+} .
$$

Clearly each Walsh function of order up to $p^{n-1}$ belongs to $\mathcal{E}_{n}\left(\mathbb{R}^{+}\right)$. The set $\mathcal{E}\left(\mathbb{R}^{+}\right)$of $p$-adic entire functions on $\mathbb{R}^{+}$is the union of all the spaces $\mathcal{E}_{n}\left(\mathbb{R}^{+}\right)$. It is clear that $\mathcal{E}\left(\mathbb{R}^{+}\right)$is dense in $L^{p}\left(\mathbb{R}^{+}\right), 1 \leq p<\infty$, and each function in $\mathcal{E}\left(\mathbb{R}^{+}\right)$is of compact support. Thus, we consider the following set of functions

$$
\mathcal{E}^{0}\left(\mathbb{R}^{+}\right)=\left\{f \in \mathcal{E}\left(\mathbb{R}^{+}\right): \operatorname{supp} f \subset \mathbb{R}^{+} \backslash\{0\}\right\} .
$$

Definition 1. Let $\mathbb{H}$ be a separable Hilbert space. A sequence $\left\{f_{k}\right\}_{k=1}^{\infty}$ in $\mathbb{H}$ is called a frame for $\mathbb{H}$ if there exist constants $A$ and $B$ with $0<A \leq B<\infty$ such that

$$
A\|f\|_{2}^{2} \leq \sum_{k=1}^{\infty}\left|\left\langle f, f_{k}\right\rangle\right|^{2} \leq B\|f\|_{2}^{2}
$$

for all $f \in \mathbb{H}$. The largest constant $A$ and the smallest constant $B$ satisfying (4) are called the upper and the lower frame bound, respectively. The sequence $\left\{f_{k}\right\}_{k=1}^{\infty}$ is called a tight frame for $\mathbb{H}$ if the upper frame bound $A$ and the lower frame bound $B$ coincide. 
In order to prove theorems to be presented in next section, we need the following results whose proofs can be found in [2].

Lemma 1. Let $\mathbb{H}$ be a Hilbert space and $\left\{f_{k}\right\}_{k=1}^{\infty}$ be a family of elements of $\mathbb{H}$. Then

$$
\sum_{k=1}^{\infty}\left|\left\langle f, f_{k}\right\rangle\right|^{2}=\|f\|_{2}^{2} \quad \text { for all } f \in \mathbb{H}
$$

if and only if

$$
f=\sum_{k=1}^{\infty}\left\langle f, f_{k}\right\rangle f_{k} \quad \text { for all } f \in \mathbb{H}
$$

Moreover, the system $\left\{f_{k}\right\}_{k=1}^{\infty}$ is a tight frame for $\mathbb{H}$ with constant 1 if either of the two above conditions is satisfied.

Lemma 2. Let $\left\{f_{k}\right\}_{k=1}^{\infty}$ be a sequence in Hilbert space $\mathbb{H}$ such that the first equality of Lemma 1 holds for all $f$ in a dense subset of $\mathbb{H}$. Then, this equality is valid for all $f$ in $\mathbb{H}$.

Theorem 1. Suppose $\left\{f_{k}\right\}_{k=1}^{\infty}$ is a tight frame with constant 1 in Hilbert space $\mathbb{H}$. If $\left\|f_{k}\right\|_{2}=1$ for all $k=1,2, \ldots$, then $\left\{f_{k}\right\}_{k=1}^{\infty}$ is an orthonormal basis for $\mathbb{H}$.

Definition 2. Let $p$ and $q$ be any two fixed positive real numbers. For any fixed positive integer $L$, let $\Psi=\left\{\psi_{1}, \psi_{2}, \ldots, \psi_{L}\right\} \subseteq L^{2}\left(\mathbb{R}^{+}\right)$. A system of the form

$$
\mathcal{G}(\Psi, p, q):=\left\{M_{m q} T_{n p} \psi_{\ell}=: w_{m q}(x) \psi_{\ell}(x \ominus n p): n, m \in \mathbb{Z}^{+}, 1 \leq \ell \leq L\right\}
$$

is called the multi-generator Gabor system, where $M_{m q} f(x)=w_{m q}(x) f(x)$ and $T_{n p} f(x)=$ $f(x \ominus n p)$ are the modulation and translation operators defined on $L^{2}\left(\mathbb{R}^{+}\right)$, respectively.

Definition 3. The Gabor system $\mathcal{G}(\Psi, p, q)$ defined by (5) is called a Gabor frame for $L^{2}\left(\mathbb{R}^{+}\right)$, if there exist constants $C$ and $D, 0<C \leq D<\infty$, such that

$$
C\|f\|_{2}^{2} \leq \sum_{\ell=1}^{L} \sum_{m \in \mathbb{Z}^{+}} \sum_{n \in \mathbb{Z}^{+}}\left|\left\langle f, M_{m q} T_{n p} \psi_{\ell}\right\rangle\right|^{2} \leq D\|f\|_{2}^{2} .
$$

We have also the following proposition (see [11]).

Proposition 1. The system $\left\{\psi(x \ominus n p): n \in \mathbb{Z}^{+}\right\}$of functions is an orthogonal system in $L^{2}\left(\mathbb{R}^{+}\right)$if and only if

$$
\sum_{n \in \mathbb{Z}^{+}}\left|\hat{\psi}\left(\xi \ominus q^{-1} n\right)\right|^{2}=|q|\|\psi\|_{2}^{2} \text { a.e. } \xi \in \mathbb{R}^{+} .
$$

Definition 4. A function $f$ defined on $\mathbb{R}^{+}$is said to be periodic with period a if $f(x \oplus n a)=$ $f(x)$ for all $x \in \mathbb{R}^{+}$and $n \in \mathbb{Z}^{+}$. 
2 Characterization OF ORTHOgONAL GABOR SYSTEMS ON POSITIVE HALF LiNE

We shall start this section by characterizing the orthogonality of Gabor systems $\mathcal{G}(\Psi, p, q)$ given by (5) in terms of the Fourier transforms of the generators.

Theorem 2. The Gabor system $\mathcal{G}(\Psi, p, q)$ given by (5) is orthogonal if and only if

$$
\begin{gathered}
\sum_{n \in \mathbb{Z}^{+}}\left|\hat{\psi}_{\ell}\left(\xi \ominus q^{-1} n\right)\right|^{2}=|q|\left\|\psi_{\ell}\right\|_{2}^{2}, \quad \text { a.e. } \xi \in \mathbb{R}^{+}, 1 \leq \ell \leq L, \\
\sum_{s \in \mathbb{N}} \hat{\psi}_{\ell}\left(\xi \oplus q^{-1} s \ominus n p\right) \overline{\hat{\psi}_{\ell}\left(\xi \oplus q^{-1} s\right)}=0, \quad \text { a.e. } \xi \in \mathbb{R}^{+},
\end{gathered}
$$

for every $n \in \mathbb{Z}^{+}, 1 \leq \ell \leq L$, and

$$
\sum_{s \in \mathbb{Z}^{+}} \hat{\psi}_{\ell}\left(\xi \oplus q^{-1} s \ominus n p\right) \overline{\hat{\psi}_{k}\left(\xi \oplus q^{-1} s\right)}=0, \quad \text { a.e. } \xi \in \mathbb{R}^{+},
$$

for every $n \in \mathbb{Z}^{+}, 1 \leq \ell, k \leq L, \ell \neq k$.

Proof. We first assume that the system $\mathcal{G}(\Psi, p, q)$ is orthogonal. Then, equation (6) is satisfied for every $\ell$, by virtue of the Proposition 1 . Moreover, using the Plancherel theorem we obtain

$$
\begin{aligned}
0=\left\langle\psi_{\ell, m, n}, \psi_{k}\right\rangle=\left\langle\hat{\psi}_{\ell, m, n}, \hat{\psi}_{k}\right\rangle & =\int_{\mathbb{R}^{+}} w_{m n p q} \overline{w_{m q}(\xi)} \hat{\psi}_{\ell}(\xi \ominus n p) \overline{\hat{\psi}_{k}(\xi)} d \xi \\
& =\sum_{s \in \mathbb{Z}^{+}} \int_{0}^{q^{-1}} w_{m n p q} \overline{w_{m q}(\xi)} \hat{\psi}_{\ell}(\xi \ominus n p) \overline{\hat{\psi}_{k}(\xi)} d \xi \\
& =w_{m n p q} \sum_{s \in \mathbb{Z}^{+}} \int_{0}^{q^{-1}} \overline{w_{m q}(\xi)} \hat{\psi}_{\ell}\left(\xi \oplus q^{-1} s \ominus n p\right) \overline{\hat{\psi}_{k}\left(\xi \oplus q^{-1} s\right)} d \xi .
\end{aligned}
$$

Now, if the series

$$
\sum_{s \in \mathbb{Z}^{+}} \int_{0}^{q^{-1}}\left|\overline{w_{m q}(\xi)} \hat{\psi}_{\ell}\left(\xi \oplus q^{-1} s \ominus n p\right) \overline{\hat{\psi}_{k}\left(\xi+q^{-1} s\right)} d \xi\right|
$$

converges, then by Beppo-Levi's theorem, we can interchange the order of integration and summation in the following integral

$$
\int_{0}^{q^{-1}} \sum_{s \in \mathbb{Z}^{+}} \overline{w_{m q}(\xi)} \hat{\psi}_{\ell}\left(\xi \oplus q^{-1} s \ominus n p\right) \overline{\hat{\psi}_{k}\left(\xi \oplus q^{-1} s\right)} d \xi .
$$

However, we observe that

$$
\begin{aligned}
\sum_{s \in \mathbb{Z}^{+}} \int_{0}^{q^{-1}}\left|\overline{w_{m q}(\xi)} \hat{\psi}_{\ell}\left(\xi \oplus q^{-1} s \ominus n p\right) \overline{\hat{\psi}_{k}\left(\xi \oplus q^{-1} s\right)} d \xi\right|=\int_{\mathbb{R}^{+}}\left|\hat{\psi}_{\ell}(\xi \ominus n p) \overline{\hat{\psi}_{k}(\xi)}\right| d \xi & \\
& \leq\left\{\int_{\mathbb{R}^{+}}\left|\hat{\psi}_{\ell}(\xi \ominus n p)\right|^{2} d \xi\right\}^{1 / 2}\left\{\int_{\mathbb{R}^{+}}\left|\overline{\hat{\psi}_{\ell^{\prime}}(\xi)}\right|^{2} d \xi\right\}^{1 / 2}<\infty
\end{aligned}
$$

Therefore, for $\ell \neq k$ and $m, n \in \mathbb{Z}^{+}$, we have 


$$
\int_{0}^{q^{-1}}\left\{\sum_{s \in \mathbb{Z}^{+}} \hat{\psi}_{\ell}\left(\xi \oplus q^{-1} s \ominus n p\right) \overline{\hat{\psi}_{k}\left(\xi \oplus q^{-1} s\right)}\right\} \overline{w_{m q}(\xi)} d \xi=0 .
$$

Since $\left\{w_{m q}(\xi): m \in \mathbb{Z}^{+}\right\}$is a basis for $L^{2}\left[0, q^{-1}\right)$ and

$$
\sum_{s \in \mathbb{Z}^{+}} \hat{\psi}_{\ell}\left(\xi \oplus q^{-1} s \ominus n p\right) \overline{\hat{\psi}_{k}\left(\xi \oplus q^{-1} s\right)}
$$

is a periodic function, so we can conclude that for $\ell=k$ and $n \in \mathbb{Z}^{+}$, we have

$$
\sum_{s \in \mathbb{Z}^{+}} \hat{\psi}_{\ell}\left(\xi \oplus q^{-1} s \ominus n p\right) \overline{\hat{\psi}_{k}\left(\xi \oplus q^{-1} s\right)}=0 \quad \text { for a.e. } \xi \in \mathbb{R}^{+} .
$$

This completes the proof of the first implication.

Now let us assume that all the three conditions are satisfied. It is easy to verify that

$$
\left\langle\psi_{\ell, m, n}, \psi_{k, m^{\prime}, n^{\prime}}\right\rangle=w_{p q\left(m-m^{\prime}\right)\left(n^{\prime}\right)}\left\langle\psi_{m-m^{\prime}, n+n^{\prime}}^{\ell} \psi^{k}\right\rangle .
$$

Moreover, Proposition 1 implies that the systems $\left\{\psi_{\ell, 0, n}: n \in \mathbb{Z}^{+}\right\}$are orthogonal for each $\ell$. Therefore to finish the proof, we can invoke Beppo-Levi's theorem, Plancherel formula and, the second and the third conditions to prove, as above, that

$$
\left\langle\psi_{\ell, m, n}, \psi_{k}\right\rangle=0 \quad \text { for } \ell \neq k, m, n \in \mathbb{Z}^{+} .
$$

This completes the proof.

To prove the completeness of Gabor systems $\mathcal{G}(\Psi, p, q)$ in $L^{2}\left(\mathbb{R}^{+}\right)$when $|p q|=1$, we set

$$
W_{m}^{\ell}=\overline{\operatorname{span}}\left\{\psi_{\ell, m, n}: n \in \mathbb{Z}^{+}\right\}, \quad 1 \leq \ell \leq L, m \in \mathbb{Z}^{+} .
$$

Assume that the Gabor systems $\mathcal{G}(\Psi, p, q)$ given by (5) are orthogonal in $L^{2}\left(\mathbb{R}^{+}\right)$and let $P_{m}^{\ell}$ denote the orthogonal projection onto the space $W_{m}^{\ell}$, that is

$$
P_{m}^{\ell} f(x)=\sum_{n \in \mathbb{Z}^{+}}\left\langle f, \psi_{\ell, m, n}\right\rangle \psi_{\ell, m, n}(x) \text { for every } f \in L^{2}\left(\mathbb{R}^{+}\right) .
$$

Then, we have

$$
\begin{aligned}
\left\langle\hat{f}, \hat{\psi}_{\ell, m, n}\right\rangle & =\int_{\mathbb{R}^{+}} \hat{f}(\xi) w_{n q}(\xi) \overline{w_{m n p q}} \overline{\hat{\psi}_{\ell}(\xi \ominus m p)} d \xi=\sum_{s \in \mathbb{Z}^{+}} \int_{0}^{q^{-1}} w_{n q}(\xi) \hat{f}(\xi) \overline{\hat{\psi}_{\ell}(\xi \ominus m p)} d \xi \\
& =\sum_{s \in \mathbb{Z}^{+}} \int_{0}^{q^{-1}} w_{n}\left(\xi \oplus q^{-1} s\right) \hat{f}\left(\xi \oplus q^{-1} s\right) \overline{\hat{\psi}_{\ell}\left(\xi \oplus q^{-1} s \ominus m p\right)} d \xi .
\end{aligned}
$$

Note that

$$
\begin{gathered}
\sum_{s \in \mathbb{Z}^{+}} \int_{0}^{q^{-1}}\left|w_{n q}(\xi) \hat{f}\left(\xi \oplus q^{-1} s\right) \overline{\hat{\psi}_{\ell}\left(\xi \oplus q^{-1} s \ominus m p\right)}\right| d \xi=\int_{\mathbb{R}^{+}}\left|\hat{f}(\xi) \overline{\hat{\psi}_{\ell}(\xi \ominus m p)}\right| d \xi \\
\quad \leq\left\{\int_{\mathbb{R}^{+}}|\hat{f}(\xi)|^{2} d \xi\right\}^{1 / 2}\left\{\int_{\mathbb{R}^{+}}\left|\hat{\psi}_{\ell}(\xi \ominus m p)\right|^{2} d \xi\right\}^{1 / 2}=\|\hat{f}\|_{2}\|\hat{\psi}\|_{2}<\infty .
\end{gathered}
$$


Therefore, we can use the Beppo-Levi's theorem to obtain

$$
\left\langle\hat{f}, \hat{\psi}_{\ell, m, n}\right\rangle=\int_{0}^{q^{-1}}\left\{\sum_{s \in \mathbb{Z}^{+}} \hat{f}\left(\xi \oplus q^{-1} s\right) \overline{\hat{\psi}_{\ell}\left(\xi \oplus q^{-1} s \ominus m p\right)}\right\} w_{n q}(\xi) d \xi .
$$

Clearly, these are the Fourier coefficients of the periodic function

$$
\sum_{s \in \mathbb{Z}^{+}} \hat{f}\left(\xi \oplus q^{-1} s\right) \overline{\hat{\psi}_{\ell}\left(\xi \oplus q^{-1} s \ominus m p\right)}
$$

thus, we can write

$$
\sum_{s \in \mathbb{Z}^{+}} \hat{f}\left(\xi \oplus q^{-1} s\right) \overline{\hat{\psi}_{\ell}\left(\xi \oplus q^{-1} s \ominus m p\right)}=|q| \sum_{n \in \mathbb{Z}^{+}}\left\langle\hat{f}, \hat{\psi}_{\ell, m, n}\right\rangle \overline{w_{n q}(\xi)}
$$

Multiplying both sides of the above identity by $\hat{\psi}_{\ell}(\xi \ominus m p)$, we obtain the desired expression for $P_{m}^{\ell}$ as

$$
\widehat{P_{m}^{\ell} f}(\xi)=\frac{1}{|p|} \sum_{s \in \mathbb{Z}^{+}} \hat{f}\left(\xi \oplus p^{-1} s\right) \overline{\hat{\psi}_{\ell}\left(\xi \oplus p^{-1} s \ominus m q\right)} \hat{\psi}_{\ell}(\xi \ominus m q) .
$$

Theorem 3. Let $\Psi=\left\{\psi_{1}, \psi_{2}, \ldots, \psi_{L}\right\} \subseteq L^{2}(K)$ and $p, q \in \mathbb{R}^{+} \backslash\{0\}$ be given. If $|p q|=1$ and the functions $\psi_{1}, \psi_{2}, \ldots, \psi_{L}$ satisfy the following three conditions:

$$
\begin{gathered}
\sum_{m \in \mathbb{Z}^{+}}\left|\hat{\psi}_{\ell}\left(\xi \ominus q^{-1} m\right)\right|^{2}=|q|\left\|\psi_{\ell}\right\|_{2}^{2}, \quad \text { a.e. } \xi \in \mathbb{R}^{+}, 1 \leq \ell \leq L, \\
\sum_{s \in \mathbb{Z}^{+}} \hat{\psi}_{\ell}\left(\xi \oplus q^{-1} s \ominus m p\right) \overline{\hat{\psi}_{\ell}\left(\xi \oplus q^{-1} s\right)}=0, \quad \text { a.e. } \xi \in \mathbb{R}^{+},
\end{gathered}
$$

for every $m \in \mathbb{Z}^{+}, 1 \leq \ell \leq L$, and

$$
\sum_{s \in \mathbb{Z}^{+}} \hat{\psi}_{\ell}\left(\xi \oplus q^{-1} s \ominus m p\right) \overline{\hat{\psi}_{k}\left(\xi \oplus q^{-1} s\right)}=0, \quad \text { a.e. } \xi \in \mathbb{R}^{+},
$$

for every $m \in \mathbb{Z}^{+}, 1 \leq \ell, k \leq L, \ell \neq k$. Then, the Gabor system $\mathcal{G}(\Psi, p, q)$ as defined in (5) is complete in $L^{2}\left(\mathbb{R}^{+}\right)$.

Proof. To prove the result, it is sufficient to prove that

$$
\sum_{\ell=1}^{L} \sum_{m \in \mathbb{Z}^{+}} \widehat{P_{m}^{\ell}} f(\xi)=\left(\sum_{\ell=1}^{L}\left\|\psi_{\ell}\right\|_{2}^{2}\right) \hat{f}(\xi) \quad \text { a.e. } \xi \in \mathbb{R}^{+},
$$

and

$$
\lim _{M \rightarrow \infty}\left\|\sum_{\ell=1}^{L} \sum_{|m| \leq M} \widehat{P_{m}^{\ell}} f\right\|_{2}=\left(\sum_{\ell=1}^{L}\left\|\psi_{\ell}\right\|_{2}^{2}\right)\|\hat{f}\|_{2}
$$

hold for every $f \in L^{2}\left(\mathbb{R}^{+}\right)$. In view of Lemma 1 and Lemma 2 , it is enough to verify that the equalities (11) and (12) hold for all $f \in \mathcal{E}^{0}$. In Theorem 2, we have already shown that the system $\mathcal{G}(\Psi, p, q)$ given by (5) is orthogonal in $L^{2}\left(\mathbb{R}^{+}\right)$; hence by applying (7) to the projections $P_{m}^{\ell}$, we can write

$$
\sum_{\ell=1}^{L} \sum_{m \in \mathbb{Z}^{+}} \widehat{P_{m}^{\ell}} f(\xi)=\frac{1}{|q|} \sum_{\ell=1}^{L} \sum_{m \in \mathbb{Z}^{+}}\left\{\sum_{s \in \mathbb{Z}^{+}} \hat{f}\left(\xi \oplus q^{-1} s\right) \overline{\psi_{\ell}\left(\xi \oplus q^{-1} s \ominus m p\right)} \hat{\psi}_{\ell}(\xi \ominus m p)\right\}
$$




$$
\begin{aligned}
& =\frac{1}{|q|} \sum_{\ell=1}^{L} \sum_{m \in \mathbb{Z}^{+}} \hat{f}(\xi)\left|\hat{\psi}_{\ell}(\xi \ominus m p)\right|^{2} \\
& \quad+\frac{1}{|q|} \sum_{\ell=1}^{L} \sum_{m \in \mathbb{Z}^{+}} \sum_{s \in \mathbb{Z}^{+}} \hat{f}\left(\xi \oplus q^{-1} s\right) \overline{\psi_{\ell}\left(\xi \oplus q^{-1} s \ominus m p\right)} \hat{\psi}_{\ell}(\xi \ominus m p) \\
& =\frac{1}{|q|} \hat{f}(\xi) \sum_{\ell=1}^{L} \sum_{m \in \mathbb{Z}^{+}}\left|\hat{\psi}_{\ell}(\xi \ominus m p)\right|^{2} \\
& \quad+\frac{1}{|q|} \sum_{\ell=1}^{L} \sum_{s \in \mathbb{Z}^{+}} \hat{f}\left(\xi \oplus q^{-1} s\right) \sum_{m \in \mathbb{Z}^{+}} \frac{\psi_{\ell}\left(\xi \oplus q^{-1} s \ominus m p\right)}{\psi_{\ell}}(\xi \ominus m p) \\
& =\hat{f}(\xi)\left(\sum_{\ell=1}^{L}\left\|\psi_{\ell}\right\|_{2}^{2}\right) .
\end{aligned}
$$

Here we have used our assumption on the functions $\psi_{\ell}$, i.e., equations (8)-(10) and the fact $|p q|=1$. The change in the order of summation is valid since $f \in \mathcal{E}^{0}\left(\mathbb{R}^{+}\right)$, which implies that the sum over $s \in \mathbb{Z}^{+}$is finite.

In order to prove the relation (12), we use the fact that $P_{m}^{\ell}$ 's are mutually orthogonal, so we have

$$
\left\|\sum_{\ell=1}^{L} \sum_{|m| \leq M} \widehat{P_{m}^{\ell}} f\right\|_{2} \leq\left(\sum_{\ell=1}^{L}\left\|\psi_{\ell}\right\|_{2}^{2}\right)^{1 / 2}\|\hat{f}\|_{2} \quad \text { for every } M>0 .
$$

Moreover, the orthogonality of $P_{m}^{\ell}$ 's implies that

$$
\left\|\sum_{\ell=1}^{L} \sum_{|m| \leq M} \widehat{P_{m}^{\ell}} f\right\|_{2}=\left(\sum_{\ell=1}^{L} \sum_{|m| \leq M}\left\|\widehat{P_{m}^{\ell} f}\right\|_{2}^{2}\right)^{1 / 2}
$$

is an increasing sequence bounded by $\left(\sum_{\ell=1}^{L}\left\|\psi_{\ell}\right\|_{2}^{2}\right)^{1 / 2}\|\hat{f}\|_{2}$. Therefore, we have

$$
\lim _{M \rightarrow \infty}\left\|\sum_{\ell=1}^{L} \sum_{|m| \leq M} \widehat{P_{m}^{\ell}} f\right\|_{2} \leq\left(\sum_{\ell=1}^{L}\left\|\psi_{\ell}\right\|_{2}^{2}\right)^{1 / 2}\|\hat{f}\|_{2} .
$$

On the other hand, by Fatou's lemma we have

$$
\lim _{M \rightarrow \infty}\left\|\sum_{\ell=1}^{L} \sum_{|m| \leq M} \widehat{P_{m}^{\ell}} f\right\|_{2} \geq\left(\sum_{\ell=1}^{L}\left\|\psi_{\ell}\right\|_{2}^{2}\right)^{1 / 2}\|\hat{f}\|_{2} .
$$

Combining the above inequalities, we get the desired result.

As a consequence of the above theorem, we get following characterization of tight Gabor frames on positive half line.

Corollary 1. Let $p, q \in \mathbb{R}^{+} \backslash\{0\}$ be given. Suppose $\Psi=\left\{\psi_{1}, \psi_{2}, \ldots, \psi_{L}\right\} \subseteq L^{2}\left(\mathbb{R}^{+}\right)$be such that $\sum_{\ell=1}^{L}\left\|\psi_{\ell}\right\|_{2}^{2}=1$. Then with the assumptions of Theorem 3, the system $\mathcal{G}(\Psi, p, q)$ given by (5) constitutes a tight frame with constant 1 for $L^{2}\left(\mathbb{R}^{+}\right)$. 
By combining Corollary 1 with Theorem 1, we can obtain the following characterization for Gabor systems generated by a single function.

Theorem 4. Let $\psi \in L^{2}\left(\mathbb{R}^{+}\right)$and $p, q \in \mathbb{R}^{+} \backslash\{0\}$ such that $|p q|=1$. Then the $\operatorname{system} \mathcal{G}(\Psi, p, q)$ is a tight frame with constant 1 if and only if the following equations hold:

$$
\begin{gathered}
\sum_{m \in \mathbb{Z}^{+}}\left|\hat{\psi}\left(\xi \ominus q^{-1} m\right)\right|^{2}=|q|, \quad \text { a.e. } \xi \in \mathbb{R}^{+}, \\
\sum_{s \in \mathbb{Z}^{+}} \hat{\psi}\left(\xi \oplus q^{-1} s \ominus m p\right) \overline{\hat{\psi}\left(\xi \oplus q^{-1} s\right)}=0, \quad \text { a.e. } \xi \in \mathbb{R}^{+}, m \in \mathbb{Z}^{+} .
\end{gathered}
$$

\section{CHARACTERIZATION OF TIGHT GABOR SYSTEMS ON POSITIVE HALF LINE}

Theorem 5. The Gabor system $\mathcal{G}(\Psi, p, q)$ given by (5) is a tight frame for $L^{2}\left(\mathbb{R}^{+}\right)$if and only if it satisfies the two conditions:

$$
\sum_{\ell=1}^{L} \sum_{n \in \mathbb{Z}^{+}}|\hat{\psi}(\xi \ominus n p)|^{2}=|q|, \quad \text { a.e. } \xi \in \mathbb{R}^{+}
$$

and

$$
\sum_{\ell=1}^{L} \sum_{n \in \mathbb{Z}^{+}} \hat{\psi}(\xi \ominus n p) \overline{\hat{\psi}\left(\xi \oplus q^{-1} m \ominus n p\right)}=0, \quad \text { a.e. } \xi \in \mathbb{R}^{+}, m \neq 0 .
$$

Proof. Since the set $\mathcal{E}^{0}\left(\mathbb{R}^{+}\right)=\left\{f \in \mathcal{E}\left(\mathbb{R}^{+}\right)\right.$: supp $\left.\hat{f} \subset \mathbb{R}^{+} \backslash\{0\}\right\}$ is dense in $L^{2}\left(\mathbb{R}^{+}\right)$. Therefore, by Lemma 2, it suffices to show that (13) and (14) imply that the Gabor system $\mathcal{G}(\Psi, p, q)$ is a tight frame for $L^{2}\left(\mathbb{R}^{+}\right)$for all $f \in \mathcal{E}^{0}$.

For every $n \in \mathbb{Z}^{+}$, we define

$$
F(\xi)=F_{n}^{\ell}(\xi)=\hat{f}(\xi \ominus n p) \overline{\hat{\psi}_{\ell}(\xi)} .
$$

Then,

$$
\begin{aligned}
\hat{F}(m p)=\int_{\mathbb{R}^{+}} F(\xi) \overline{w_{m p}(\xi)} d \xi & =\sum_{s \in \mathbb{Z}^{+}} \int_{0}^{p^{-1}} F(\xi) \overline{w_{m p}(\xi)} d \xi \\
& =\int_{0}^{p^{-1}} \overline{w_{m p}(\xi)}\left(\sum_{s \in \mathbb{Z}^{+}} F\left(\xi \oplus p^{-1} s\right)\right) d \xi
\end{aligned}
$$

The interchange of the order of summation and integration is justified by the fact that $f \in \mathcal{E}^{0}\left(\mathbb{R}^{+}\right)$is compactly supported and so is the function $F$ and, consequently the sum over $s$ is finite. Therefore, we can write

$$
\begin{aligned}
|q| \sum_{m \in \mathbb{Z}^{+}}|\hat{F}(q m)|^{2} & =\left\|\sum_{s \in \mathbb{Z}^{+}} F\left(\xi \oplus q^{-1} s\right)\right\|_{L^{2}(\mathfrak{D})}^{2} \\
& =\int_{\mathfrak{D}}\left(\sum_{s \in \mathbb{Z}^{+}} F\left(\xi \oplus q^{-1} s\right)\right) \overline{\left(\sum_{m \in \mathbb{Z}^{+}} F\left(\xi \oplus q^{-1} m\right)\right)} d \xi \\
& =\int_{\mathbb{R}^{+}}\left(\sum_{s \in \mathbb{Z}^{+}} F\left(\xi \oplus q^{-1} s\right) \overline{F(\xi)} d \xi .\right.
\end{aligned}
$$


Now from the definition of $F$, we obtain the following equality:

$$
\begin{aligned}
&|q| \sum_{m \in \mathbb{Z}^{+}} \mid\left.\int_{\mathbb{R}^{+}} \hat{f}(\xi \oplus n p) \overline{\hat{\psi}_{\ell}(\xi)} \overline{w_{m q}(\xi)} d \xi\right|^{2} \\
& \quad=\int_{\mathbb{R}^{+}} \overline{\hat{f}(\xi \oplus n p)} \hat{\psi}_{\ell}(\xi)\left(\sum_{s \in \mathbb{Z}^{+}} \hat{f}\left(\xi \oplus q^{-1} s \oplus n p\right) \overline{\hat{\psi}_{\ell}\left(\xi \oplus q^{-1} s\right)}\right) d \xi .
\end{aligned}
$$

Further, by using Plancherel theorem and previous equality, we obtain

$$
\begin{aligned}
\sum_{\ell=1}^{L} & \sum_{m \in \mathbb{Z}^{+}} \sum_{n \in \mathbb{Z}^{+}}\left|\left\langle f, \psi_{\ell, m, n}\right\rangle\right|^{2}=\sum_{\ell=1}^{L} \sum_{m \in \mathbb{Z}^{+}} \sum_{n \in \mathbb{Z}^{+}}\left|\left\langle\hat{f}, \hat{\psi}_{\ell, m, n}\right\rangle\right|^{2} \\
& =\sum_{\ell=1}^{L} \sum_{m \in \mathbb{Z}^{+}} \sum_{n \in \mathbb{Z}^{+}} \int_{\mathbb{R}^{+}}\left|\hat{f}(\xi) \overline{\hat{\psi}_{\ell}(\xi \ominus n p)} \overline{w_{m q}(\xi)} d \xi\right|^{2} \\
& =\sum_{\ell=1}^{L} \sum_{m \in \mathbb{Z}^{+}} \sum_{n \in \mathbb{Z}^{+}} \int_{\mathbb{R}^{+}}\left|\hat{f}(\xi \oplus n p) \overline{\hat{\psi}_{\ell}(\xi)} \overline{w_{m q}(\xi)} d \xi\right|^{2} \\
& =\sum_{\ell=1}^{L} \sum_{n \in \mathbb{Z}^{+}} \frac{1}{|q|} \int_{\mathbb{R}^{+}} \overline{\hat{f}(\xi \oplus n p)} \hat{\psi}_{\ell}(\xi)\left(\sum_{s \in \mathbb{Z}^{+}} \hat{f}\left(\xi \oplus q^{-1} s \oplus n p\right) \overline{\hat{\psi}_{\ell}\left(\xi \oplus q^{-1} s\right)}\right) d \xi \\
& =\sum_{\ell=1}^{L} \sum_{n \in \mathbb{Z}^{+}} \frac{1}{|q|} \int_{\mathbb{R}^{+}}|\hat{f}(\xi \oplus n p)|^{2}\left|\hat{\psi}_{\ell}(\xi)\right|^{2} d \xi \\
& +\sum_{\ell=1}^{L} \sum_{n \in \mathbb{Z}^{+}} \frac{1}{|q|} \int_{\mathbb{R}^{+}} \overline{\hat{f}(\xi \oplus n p)} \hat{\psi}_{\ell}(\xi)\left(\sum_{m \in \mathbb{N}} \hat{f}\left(\xi \oplus q^{-1} m \oplus n p\right) \overline{\hat{\psi}_{\ell}\left(\xi \oplus q^{-1} m\right)}\right) d \xi \\
& \left.=\sum_{\ell=1}^{L} \sum_{n \in \mathbb{N}_{0}} \frac{1}{|q|} \int_{\mathbb{R}^{+}}|\hat{f}(\xi)|^{2} \mid \hat{\psi}_{\ell}(\xi \ominus n p)\right)\left.\right|^{2} d \xi \\
& +\sum_{\ell=1}^{L} \sum_{n \in \mathbb{Z}^{+}} \frac{1}{|q|} \int_{\mathbb{R}^{+}} \overline{\hat{f}(\xi)} \hat{\psi}_{\ell}(\xi \ominus n p)\left(\sum_{m \in \mathbb{N}} \hat{f}\left(\xi \oplus q^{-1} m\right) \overline{\hat{\psi}_{\ell}\left(\xi \oplus q^{-1} m \ominus n p\right)}\right) d \xi \\
& =I_{1}+I_{2}
\end{aligned}
$$

where

$$
\left.I_{1}=\sum_{\ell=1}^{L} \sum_{n \in \mathbb{Z}^{+}} \frac{1}{|q|} \int_{\mathbb{R}^{+}}|\hat{f}(\xi)|^{2} \mid \hat{\psi}_{\ell}(\xi \ominus n p)\right)\left.\right|^{2} d \xi
$$

and

$$
I_{2}=\sum_{\ell=1}^{L} \sum_{n \in \mathbb{Z}^{+}} \frac{1}{|q|} \int_{\mathbb{R}^{+}} \overline{\hat{f}(\xi)} \hat{\psi}_{\ell}(\xi \ominus n p)\left(\sum_{m \in \mathbb{N}} \hat{f}\left(\xi \oplus q^{-1} m\right) \overline{\hat{\psi}_{\ell}\left(\xi \oplus q^{-1} m \ominus n p\right)}\right) d \xi
$$

Now, in order to justify the multiplication that show the second summand $I_{2}$ to interchange the summation and integration, we should prove the following fact.

Fact. For $f \in \mathcal{E}^{0}\left(\mathbb{R}^{+}\right), 1 \leq \ell \leq L$ and $\psi_{\ell} \in L^{2}\left(\mathbb{R}^{+}\right)$, we have

$$
\left.\sum_{n \in \mathbb{Z}^{+}} \int_{\mathbb{R}^{+}} \mid \hat{f}(\xi \oplus n p)\right)|| \hat{\psi}_{\ell}(\xi) \mid\left(\sum_{m \in \mathbb{N}}\left|\hat{f}\left(\xi \oplus q^{-1} m \oplus n p\right)\right|\left|\hat{\psi}_{\ell}\left(\xi \oplus q^{-1} m\right)\right|\right) d \xi<\infty
$$


This is an easy consequence of the following inequality:

$$
\left|\hat{\psi}_{\ell}(\xi)\right|\left|\hat{\psi}_{\ell}\left(\xi \oplus q^{-1} m\right)\right| \leq\left|\hat{\psi}_{\ell}(\xi)\right|^{2} \oplus\left|\hat{\psi}_{\ell}\left(\xi \oplus q^{-1} m\right)\right|^{2}
$$

and the fact that $f$ has compact support and is bounded.

By the above Fact, we can rewrite $I_{2}$ as

$$
\begin{aligned}
I_{2} & =\sum_{\ell=1}^{L} \sum_{n \in \mathbb{Z}^{+}} \frac{1}{|q|} \int_{\mathbb{R}^{+}} \overline{\hat{f}(\xi)} \hat{\psi}_{\ell}(\xi \ominus n p)\left(\sum_{m \in \mathbb{N}} \hat{f}\left(\xi \oplus q^{-1} m\right) \overline{\hat{\psi}_{\ell}\left(\xi \oplus q^{-1} m \ominus n p\right)}\right) d \xi \\
& =\int_{\mathbb{R}^{+}} \overline{\hat{f}(\xi)} \sum_{m \in \mathbb{N}} \hat{f}\left(\xi \oplus q^{-1} m\right) G_{m}(\xi) d \xi,
\end{aligned}
$$

where

$$
G_{m}(\xi)=\frac{1}{|q|} \sum_{\ell=1}^{L} \sum_{n \in \mathbb{Z}^{+}} \hat{\psi}_{\ell}(\xi \ominus n p) \overline{\hat{\psi}_{\ell}\left(\xi \oplus q^{-1} m \ominus n p\right)}, \text { for } m \in \mathbb{Z}^{+} .
$$

Therefore, it is clear that if the system (5) satisfies equations (13) and (14), then

$$
\left.I_{1}=\sum_{n \in \mathbb{Z}^{+}} \frac{1}{|q|} \int_{\mathbb{R}^{+}}|\hat{f}(\xi)|^{2} \mid \hat{\psi}_{\ell}(\xi \ominus n p)\right)\left.\right|^{2} d \xi=\int_{\mathbb{R}^{+}}|\hat{f}(\xi)|^{2} d \xi=\|f\|_{2^{\prime}}^{2}
$$

and $I_{2}=0$. Hence, equation (15) becomes

$$
\sum_{\ell=1}^{L} \sum_{m \in \mathbb{Z}^{+}} \sum_{n \in \mathbb{Z}^{+}}\left|\left\langle f, \psi_{\ell, m, n}\right\rangle\right|^{2}=I_{1}+I_{2}=\|f\|_{2}^{2}
$$

which completes the proof of the fact that any Gabor system (5) satisfies (13) and (14) is a tight frame for $L^{2}\left(\mathbb{R}^{+}\right)$.

Conversely, suppose that the Gabor system defined by (5) is a tight frame for $L^{2}\left(\mathbb{R}^{+}\right)$, i.e.,

$$
\sum_{\ell=1}^{L} \sum_{m \in \mathbb{Z}^{+}} \sum_{n \in \mathbb{Z}^{+}}\left|\left\langle f, \psi_{\ell, m, n}\right\rangle\right|^{2}=\|f\|_{2}^{2}, \quad \text { for all } f \in L^{2}\left(\mathbb{R}^{+}\right) .
$$

Clearly, our condition holds in particular for all functions $f \in \mathcal{E}^{0}\left(\mathbb{R}^{+}\right)$. Therefore, by varying $f$ in $\mathcal{E}^{0}\left(\mathbb{R}^{+}\right)$, we see from above that $I_{1}<+\infty$ if and only if $G_{0}(\xi)=\frac{1}{|q|} \sum_{\ell=1}^{L} \sum_{n \in \mathbb{Z}^{+}}\left|\hat{\psi}_{\ell}(\xi \ominus n p)\right|^{2}$ is locally integrable in $\mathbb{R}^{+}$. Thus, almost every point in $\mathbb{R}^{+}$is a regular point of the integral of $G_{0}(\xi)$. This means that if $\xi_{0}$ is such a point, then

$$
\lim _{m \rightarrow \infty} \int_{\xi-x \in I_{m}(x)} G_{0}(\xi) d \xi=G_{0}\left(\xi_{0}\right)
$$

Let us fix $M>0$ and choose $f^{\prime}$ be a function such that

$$
\hat{f}^{\prime}(\xi)=w_{m}\left(\xi-\xi_{0}\right), m \geq M
$$

where $w_{m}\left(\xi-\xi_{0}\right)$ are Walsh functions of $\xi_{0}+I_{m}(x)$. Recall the decomposition, we have used in the proof of first implication, we have

$$
I^{\prime}=I_{1}^{\prime}+I_{2}^{\prime}
$$


Therefore, $I^{\prime}=\left\|f^{\prime}\right\|^{2}=\left\|\hat{f}^{\prime}\right\|^{2}=1$ and

$$
1=\int_{\tilde{\xi}_{0}-x \in I_{m}(x)} G_{0}(\xi) d \xi+I_{2}^{\delta}
$$

as $M \rightarrow \infty$. Now, if we can show that $I_{2}^{\prime} \rightarrow 0$ as $m \rightarrow \infty$, then (since $\xi_{0}$ is a regular point of the integral of $G_{0}(\xi)$ ), we have

$$
1=\lim _{n \rightarrow 0} \int_{\xi-x \in I_{n}(x)} G_{0}(\xi) d \xi+I_{2}^{\prime}=G_{0}\left(\xi_{0}\right)+\lim _{n \rightarrow 0} I_{2}^{\prime}=G_{0}\left(\xi_{0}\right)
$$

and hence (13) is satisfied by the Gabor system (5). To do this, we argue as we do in the proof of the Fact, we see that $\left|I_{2}^{\prime}\right|$ is dominated by the sum of two terms:

$$
\left.\frac{1}{|q|} \sum_{\ell=1}^{L} \sum_{n \in \mathbb{Z}^{+}} \int_{\mathbb{R}^{+}} \sum_{m \in \mathbb{N}}\left|\hat{f}^{\prime}(\xi)\right|\left|\hat{f}^{\prime}\left(\xi \oplus q^{-1} m\right)\right| \mid \hat{\psi}_{\ell}(\xi \ominus n p)\right)\left.\right|^{2} d \xi
$$

and

$$
\left.\frac{1}{|q|} \sum_{\ell=1}^{L} \sum_{n \in \mathbb{Z}^{+}} \int_{\mathbb{R}^{+}} \sum_{m \in \mathbb{N}}\left|\hat{f}^{\prime}(\xi)\right|\left|\hat{f}^{\prime}\left(\xi \oplus q^{-1} m\right)\right| \mid \hat{\psi}_{\ell}\left(\xi \oplus q^{-1} m \ominus n p\right)\right)\left.\right|^{2} d \xi .
$$

Moreover, for every $m \neq 0$, we have

$$
\left|\hat{f}^{\prime}(\xi)\right|\left|\hat{f}^{\prime}\left(\xi \oplus q^{-1} m\right)\right|=0
$$

as $m \rightarrow \infty$. Thus, $I_{2}^{\prime}=0$ as $m \rightarrow \infty$ and therefore, we can conclude that $G_{0}(\xi)=1$, a.e. $\xi \in \mathbb{R}^{+}$, i.e., the equality in (13) is satisfied by the Gabor system (5)

$$
\sum_{\ell=1}^{L} \sum_{n \in \mathbb{Z}^{+}}\left|\hat{\psi}_{\ell}(\xi \ominus n p)\right|^{2}=|q|, \quad \text { a.e. } \xi \in K \text {. }
$$

This also shows that $I_{2}=0$ for all $f \in \mathcal{E}^{0}\left(\mathbb{R}^{+}\right)$, i.e.,

$$
I_{2}=\int_{\mathbb{R}^{+}} \overline{\hat{f}(\xi)} \sum_{m \in \mathbb{N}} \hat{f}\left(\xi \oplus q^{-1} m\right) G_{m}(\xi) d \xi=0 .
$$

Further, by the application of the polarization identity, we get

$$
\int_{\mathbb{R}^{+}} \overline{\hat{f}(\xi)} \sum_{m \in \mathbb{N}} \hat{h}\left(\xi \oplus q^{-1} m\right) G_{m}(\xi) d \xi=0 \text { for all } f, h \in \mathcal{E}^{0}\left(\mathbb{R}^{+}\right) .
$$

Let us fix $m_{0} \neq 0$ and choose a regular point $\xi_{0}$ of the integral of $G_{m_{0}}$ such that $\xi_{0} \neq 0$. Since $G_{0}$ is locally integrable on $\mathbb{R}^{+}$and therefore, almost every point in $\mathbb{R}^{+}$is a regular point of its integral. Let $f_{m}$ and $h_{m}$ in $\mathcal{E}^{0}\left(\mathbb{R}^{+}\right)$be functions such that

$$
\hat{f}_{m}(\xi)=w_{m}\left(\xi-\xi_{0}\right), m \geq M
$$

and

$$
\hat{h}_{m}(\xi)=w_{m}\left(\xi-q^{-1} \xi_{0}\right) .
$$

Then, in particular, we have

$$
\hat{f}_{m}(\xi) \hat{h}_{m}\left(\xi_{0} \oplus q^{-1} m_{0}\right)=\chi_{\xi-x \in I_{n}(x)}(\xi) .
$$


Let us now write (16) using the functions $f_{m}$ and $h_{m}$ :

$$
\begin{aligned}
0 & =\int_{\mathbb{R}^{+}} \overline{\hat{f}_{\delta}(\xi)} \sum_{m \in \mathbb{N}} \hat{h}_{m}\left(\xi \oplus q^{-1} m\right) G_{m}(\xi) d \xi \\
& =\int_{\mathbb{R}^{+}} \overline{\hat{f}_{\delta}(\xi)} \hat{h}_{\delta}\left(\xi \oplus q^{-1} m_{0}\right) G_{m_{0}}(\xi) d \xi+\int_{\mathbb{R}^{+}} \overline{\hat{f}_{\delta}(\xi)} \sum_{m_{0} \neq m \in \mathbb{N}} \hat{h}_{\delta}\left(\xi \oplus q^{-1} m\right) G_{m}(\xi) d \xi \\
& =\int_{\xi-x \in I_{m}(x)} G_{m_{0}}(\xi) d \xi+J^{\prime} .
\end{aligned}
$$

By examining the term $J^{\prime}$ very closely, we see as before, that as $m \rightarrow \infty$, it is equal to zero. Therefore, by employing the fact that $\xi_{0}$ was chosen to be a regular point of $G_{m_{0}}(\xi)$, we obtain (14) as:

$$
\lim _{m \rightarrow \infty} \int_{\xi-x \in I_{m}(x)} G_{m_{0}}(\xi) d \xi=0
$$

which implies that

$$
\sum_{n \in \mathbb{Z}^{+}} \hat{\psi}_{\ell}(\xi \ominus n p) \overline{\hat{\psi}_{\ell}\left(\xi \oplus q^{-1} m \ominus n p\right)}=0, \quad \text { a.e. } \xi \in \mathbb{R}^{+}, m \neq 0 .
$$

This completes the proof of the theorem.

Theorem 6. The Gabor system $\mathcal{G}(\Psi, p, q)$ given by (5) is an orthonormal basis for $L^{2}\left(\mathbb{R}^{+}\right)$if and only if it satisfies equations (13) and (14) together with $\left\|\psi_{\ell}\right\|_{2}=1,1 \leq \ell \leq L$.

Proof. Suppose that the Gabor system $\mathcal{G}(\Psi, p, q)$ given by (5) is an orthonormal basis for $L^{2}\left(\mathbb{R}^{+}\right)$, then clearly it is a tight frame and hence by Theorem 5 , it follows that equations (13) and (14) are satisfied. Further, the orthonormality of the system implies that $\left\|\psi_{\ell}\right\|_{2}=1$. Conversely, in view of Theorem 5, equations (13) and (14) imply that the Gabor system (5) is a tight frame for $L^{2}\left(\mathbb{R}^{+}\right)$. Since $\left\|\psi_{\ell, m, n}\right\|_{2}=\|g\|_{2}$, so by Theorem 1 , we conclude that the Gabor system given by (5) is an orthonormal basis for $L^{2}\left(\mathbb{R}^{+}\right)$.

\section{REFERENCES}

[1] Ahmad O., Shah F.A., Sheikh N.A. Gabor frames on non-Archimedean fields. Int. J. Geom. Methods Mod. Phys. 2018, 15, 1850079 (17 pages). doi:10.1142/S0219887818500792

[2] Christensen O. An Introduction to Frames and Riesz Bases. Birkhäuser, Boston, 2016.

[3] Daubechies I., Grossmann A., Meyer Y. Painless non-orthogonal expansions. J. Math. Phys. 1986, 27 (5), $1271-$ 1283. doi:10.1063/1.527388

[4] Duffin R.J., Shaeffer A.C. A class of nonharmonic Fourier series. Trans. Amer. Math. Soc. 1952, 72, $341-366$. doi:10.2307/1990760

[5] Farkov Yu.A. Orthogonal p-wavelets on $\mathbb{R}^{+}$. In: Proc. Int. Conf. Wavelets and Splines, St. Peterburg State University, 4-26, 2005.

[6] Farkov Yu.A., Rodionov E.A. Nonstationary wavelets related to the Walsh functions. Amer. J. Comput. Math. 2012, 2, 82-87. doi:10.4236/ajcm.2012.22011

[7] Farkov Yu.A. On wavelets related to Walsh series. J. Approx. Theory 2009, 161, 259-279. doi:10.1016/j.jat.2008.10.003 
[8] Gabor D. Theory of communications. J. Inst. Elect. Engn. 1946, 93, 429-457.

[9] Gröchenig K., Janssen A.J., Kaiblinger N., Pfander G. Note on B-splines, wavelet scaling functions, and Gabor frames. IEEE Trans. Inform. Theory 2003, 49 (12), 3318-3320. doi:10.1109/TIT.2003.820022

[10] Gröchenig K. Foundation of Time-Frequency Analysis. Birkhäuser, Boston, 2001.

[11] Golubov B.I., Efimov A.V., Skvortsov V.A. Walsh Series and Transforms: Theory and Applications. Kluwer, Dordrecht, 1991.

[12] Ron A., Shen Z. Weyl-Heisenberg frames and Riesz bases in $L^{2}\left(\mathbb{R}^{d}\right)$. Duke Math. J. 199789 (2), $237-282$. doi:10.1215/S0012-7094-97-08913-4

[13] Schipp F., Wade W.R., Simon P. Walsh Series: An Introduction to Dyadic Harmonic Analysis. Adam Hilger, Bristol and New York, 1990.

[14] Shah F.A. Gabor frames on a half-line. J. Contemp. Math. Anal. 2012, 47 (5), 251-260. doi:10.3103/S1068362312050056

[15] Shah F.A., Ahmad O., Sheikh N.A. Orthogonal Gabor systems on local fields. Filomat 2017, 31 (16), $5193-5201$. doi:10.2298/FIL1716193S

[16] Shah F.A., Ahmad O. Wave packet systems on local fields. J. Geom. Phys. 2017, 120, 5-18. doi:10.1016/j.geomphys.2017.05.015

[17] Shah F.A., Ahmad O., Rahimi A. Frames Associated with Shift Invariant Spaces on Local Fields. Filomat 2018, 32 (9), 3097-3110. doi:10.2298/FIL1809097S

[18] Zhang Y. Walsh Shift-Invariant Sequences and p-adic Nonhomogeneous Dual Wavelet Frames in $L^{2}\left(\mathbb{R}^{+}\right)$. Results Math. 2019, 74, 111, 26 pp. doi:10.1007/s00025-019-1034-7

Received 13.01.2020

Revised 14.09.2020

Ахмад О., Шейх Н.А. Аослідження систем Габора на додатній півосі за допомогою перетворення Уолша-Фур'є // Карпатські матем. публ. - 2020. - Т.12, №2. - С. 468-482.

Системи Габора відіграють життєво важливу роль не тільки в сучасному гармонічному аналізі, але і в декількох областях прикладної математики, наприклад, виявлення сигналів або обробка зображень. У цій роботі ми досліджуємо системи Габора на додатній півосі за допомогою перетворення Уолша-Фур'є. Ми надаємо повну характеризацію ортогональних систем Габора на додатній півосі. Більш того, ми пропонуємо характеризацію щільних рамок та ортонормованих базисів систем Габора на додатній півосі в області Фур'є.

Ключові слова і фрази: рамка Габора, перетворення Уолша-Фур'є, ортогональність. 